\title{
Correction to: Measuring Personal Values in Cross-Cultural User-Generated Content
}

\author{
Yiting Shen, Steven R. Wilson, and Rada Mihalcea
}

\section{Correction to: \\ Chapter "Measuring Personal Values in Cross-Cultural User-Generated Content" in: I. Weber et al. (Eds.): \\ Social Informatics, LNCS 11864, https://doi.org/10.1007/978-3-030-34971-4_10}

The original version of this chapter was revised. A missing citation was added and the bibliography was updated accordingly. 